This is an electronic reprint of the original article. This reprint may differ from the original in pagination and typographic detail.

Author(s): Xayavong, L.; Smirnova, N. A.; Bender, M.; Bennaceur, Karim

Title: $\quad$ Shell-model calculation of isospin-symmetry breaking correction to superallowed Fermi beta decay

Year: $\quad 2017$

Version:

Please cite the original version:

Xayavong, L., Smirnova, N. A., Bender, M., \& Bennaceur, K. (2017). Shell-model calculation of isospin-symmetry breaking correction to superallowed Fermi beta decay. In XXIII Nuclear Physics Workshop “Marie and Pierre Curie" Essential Problems in Nuclear Physics : Proceedings (pp. 285-290). Jagiellonian University. Acta Physica Polonica B : Proceedings Supplement, 10. https://doi.org/10.5506/APhysPolBSupp.10.285

All material supplied via JYX is protected by copyright and other intellectual property rights, and duplication or sale of all or part of any of the repository collections is not permitted, except that material may be duplicated by you for your research use or educational purposes in electronic or print form. You must obtain permission for any other use. Electronic or print copies may not be offered, whether for sale or otherwise to anyone who is not an authorised user. 


\title{
SHELL-MODEL CALCULATION OF ISOSPIN-SYMMETRY BREAKING CORRECTION TO SUPERALLOWED FERMI BETA DECAY*
}

\author{
L. Xayavong ${ }^{a}$, N.A. Smirnova ${ }^{a}$, M. Bender $^{a, b}$, K. Bennaceur $^{\text {b,c }}$ \\ ${ }^{\mathrm{a}}$ CENBG (CNRS/IN2P3 - Université de Bordeaux) \\ 33175 Gradignan cedex, France

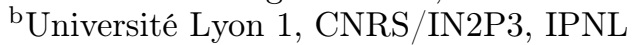 \\ 69622 Villeurbanne, France \\ ${ }^{\mathrm{c}}$ Department of Physics, University of Jyväskylä \\ P.O. Box 35 (YFL), 40014 Jyväskylä, Finland
}

(Received January 10, 2017)

\begin{abstract}
We investigate the radial-overlap part of the isospin-symmetry breaking correction to superallowed $0^{+} \rightarrow 0^{+} \beta$ decay. The $8 s d$-shell emitters with masses between $A=22$ and $A=38$ have been re-examined. The Fermi matrix element is evaluated with realistic spherical single-particle wave functions, obtained from spherical Woods-Saxon (WS) or HartreeFock (HF) potentials, fine-tuned to reproduce the experimental data on charge radii and separation energies for nuclei of interest. The elaborated adjustment procedure removes any sensitivity of the correction to a specific parametrisation of the WS potential or to various versions of the Skyrme interaction. The present results are generally in a good agreement with those already reported. At the same time, we find that the calculations with $\mathrm{HF}$ wave functions result in systematically lower values of the correction.
\end{abstract}

DOI:10.5506/APhysPolBSupp.10.285

\section{Physics of superallowed $\beta$ decay}

It has been pointed out that the superallowed $0^{+} \rightarrow 0^{+}$nuclear $\beta$ decay provides an excellent tool to probe the fundamental symmetries underlying the Standard Model of electroweak interaction, including the Conserved Vector Current (CVC) hypothesis and the unitarity of the Cabibbo-KobayashiMaskawa (CKM) quark-mixing matrix. According to the CVC hypothesis, the corrected $\mathcal{F} t$ value should relate to $G_{\mathrm{V}}$, a fundamental vector coupling

* Presented at the XXIII Nuclear Physics Workshop "Marie and Pierre Curie", Kazimierz Dolny, Poland, September 27-October 2, 2016. 
constant for a semi-leptonic decay, and thus be constant for all emitters. Traditionally, this relation is expressed as

$$
\mathcal{F} t=f t\left(1+\delta_{\mathrm{R}}^{\prime}\right)\left(1+\delta_{\mathrm{NS}}-\delta_{\mathrm{C}}\right)=\frac{K}{2 G_{\mathrm{V}}^{2}\left(1+\Delta_{\mathrm{R}}^{\mathrm{V}}\right)},
$$

where $K /(\hbar c)^{6}=2 \pi^{3} \ln (2) \hbar /\left(m_{e} c\right)^{5}=(8120.2716 \pm 0.012) \times 10^{-10} \mathrm{GeV}^{-4}$ sec, $\Delta_{\mathrm{R}}^{\mathrm{V}}, \delta_{\mathrm{R}}^{\prime}$ and $\delta_{\mathrm{NS}}$ are transition-independent, transition-dependent and nuclear structure-dependent parts of a radiative correction [1], $\delta_{\mathrm{C}}$ is an isospinsymmetry breaking correction, defined as a deviation of the Fermi matrix element squared from its model-independent value

$$
\left|M_{\mathrm{F}}\right|^{2}=\left|M_{\mathrm{F}}^{0}\right|^{2}\left(1-\delta_{\mathrm{C}}\right),
$$

with $\left|M_{\mathrm{F}}^{0}\right|=\sqrt{T(T+1)-T_{z i} T_{z f}}$.

The quantity $f t$ is determined experimentally by measuring the partial half-life, the $Q_{\mathrm{EC}}$ value and the Fermi branching ratio. The most recent survey of world data [2] finds 14 of these superallowed transitions with measured $f t$ values known to $0.1 \%$ precision or better. If the CVC holds, one can thus extract $G_{\mathrm{V}}$. By comparing it to the vector coupling constant from a muon decay, the CKM mixing matrix element between $u$ and $d$ quarks, $\left|V_{u d}\right|$ can be determined, providing a precise test of the unitarity condition of the CKM matrix.

On the theoretical side, there is still no consensus between various calculations of $\delta_{\mathrm{C}}$ (see Ref. [2] for a recent review). The present work explores the differences between shell-model calculations supplemented by WS or HF radial wave functions in comparison with the previous studies $[1,3]$.

\section{Shell-model description of the isospin correction}

Within the shell model, the Fermi matrix element of the $\beta^{+}$decay between an initial $|i\rangle$ and final $|f\rangle$ many-body states can be written as

$$
M_{\mathrm{F}}=\left\langle f\left|T_{+}\right| i\right\rangle=\sum_{\alpha} \sum_{\pi}\left\langle f\left|a_{\alpha_{n}}^{\dagger}\right| \pi\right\rangle\left\langle\pi\left|a_{\alpha_{p}}\right| i\right\rangle\left\langle\alpha_{n}\left|\hat{t}_{+}\right| \alpha_{p}\right\rangle^{\pi},
$$

where $\alpha$ denotes a full set of spherical quantum numbers of a single-particle state, $\pi$ refers to a complete set of states of an $(A-1)$ nucleus compatible with the angular momentum and parity conservation, and $\left\langle\alpha_{n}\left|\hat{t}_{+}\right| \alpha_{p}\right\rangle^{\pi}$ is the single-particle matrix element of the isospin operator ${ }^{1}$ between proton and neutron radial wave functions

$$
\left\langle\alpha_{n}\left|\hat{t}_{+}\right| \alpha_{p}\right\rangle^{\pi}=\Omega_{\alpha}^{\pi}=\int_{0}^{\infty} R_{\alpha_{n}}^{\pi}(r) R_{\alpha_{p}}^{\pi}(r) r^{2} \mathrm{~d} r .
$$

\footnotetext{
${ }^{1}$ We neglect the radial excitations as was pointed out by Miller and Schwenk [4].
} 
For harmonic oscillator functions, the latter is equal to one. To overcome this artefact, we have to replace the harmonic oscillator radial wave functions by realistic radial wave functions obtained from a spherically-symmetric WS or the self-consistent HF potential. A sum over intermediate states $\pi$ in Eq. (2.1) allows us to go beyond the closure approximation and take into account the dependence of $\Omega_{\alpha}^{\pi}$ on the excitation energies of the intermediate states, $E_{\pi}$. For each $E_{\pi}$, we fine-tune our potential so that the individual energies of valence space orbitals match experimental proton or neutron separation energies.

Substituting Eq. (2.1) into Eq. (1.2), we obtain a suitable expression for $\delta_{\mathrm{C}}$, as a sum of two terms, $\delta_{\mathrm{C}} \approx \delta_{\mathrm{RO}}+\delta_{\mathrm{IM}}$. The first term, $\delta_{\mathrm{RO}}$, is the contribution due to the deviation from unity of the overlap integral between the radial parts of the proton and neutron single-particle wave functions. It is called a radial-overlap correction and can be expressed as

$$
\delta_{\mathrm{RO}}=\frac{2}{M_{\mathrm{F}}^{0}} \sum_{\alpha} \sum_{\pi}\left\langle f\left\|a_{\alpha_{n}}^{\dagger}\right\| \pi\right\rangle^{T}\left\langle i\left\|a_{\alpha_{p}}^{\dagger}\right\| \pi\right\rangle^{T}\left(1-\Omega_{\alpha}^{\pi}\right),
$$

where the reduced matrix elements, $\left\langle f\left\|a_{\alpha_{n}}^{\dagger}\right\| \pi\right\rangle^{T}$ and $\left\langle i\left\|a_{\alpha_{p}}^{\dagger}\right\| \pi\right\rangle^{T}$ are related to the spectroscopic amplitudes [3] for neutron and proton pick-up respectively. The superscript $T$ means that these quantities are computed with an isospin-invariant effective interaction.

The other term, $\delta_{\mathrm{IM}}$, is the so-called isospin-mixing correction $[5,6]$, arising due to the isospin-mixing in many-body configurations of the initial and final states. It is obtained from the shell-model diagonalisation using a charge-dependent two-body effective interaction and is expressed as

$$
\delta_{\mathrm{IM}}=\frac{2}{M_{\mathrm{F}}^{0}} \sum_{\alpha}\left[\left\langle f\left|a_{\alpha_{n}}^{\dagger} a_{\alpha_{p}}\right| i\right\rangle^{T}-\left\langle f\left|a_{\alpha_{n}}^{\dagger} a_{\alpha_{p}}\right| i\right\rangle\right] .
$$

In this work, we focus only on the radial-overlap correction, calculating it within the shell model in combination with the realistic radial wave functions obtained from a WS or Skyrme-HF single-particle potential.

\section{Results for $\delta_{\mathrm{RO}}$ and discussions}

The radial-overlap correction, $\delta_{\mathrm{RO}}$ has been evaluated using the procedures outlined in the previous section. For this study, we choose only $s d$-shell emitters which are well-described by the so-called universal sd interactions — USD, USDA/B [7,8]. They include ${ }^{22} \mathrm{Mg},{ }^{26} \mathrm{Al},{ }^{26} \mathrm{Si},{ }^{30} \mathrm{~S},{ }^{34} \mathrm{Cl},{ }^{34} \mathrm{Ar},{ }^{38} \mathrm{~K}$ and ${ }^{38} \mathrm{Ca}$. Six of these transitions are used to deduce the most precise $\mathcal{F} t$ value, while the decays of ${ }^{26} \mathrm{Si}$ and ${ }^{30} \mathrm{~S}$ are expected to be measured with an improved precision in future radioactive-beam facilities. 
The shell-model calculations have been performed in the full $s d$ shell, using NuShellX@MSU code [9]. To get convergence, up to 100 intermediate states of each spin have been taken into account in Eq. (2.3).

Figure 1 shows the results for $\delta_{\mathrm{RO}}$ obtained with either WS or HF singleparticle wave functions. The WS results have been computed using two different parametrisations. One of them is that of Schwierz, Wiedenhöver and Volya (SWV) [10], while the other is that of Bohr and Mottelson [11], modified as proposed in Ref. [12] and denoted as $\mathrm{BM}_{\mathrm{m}}$. It is important to note that the Coulomb and the charge-symmetric isovector terms are the only sources of the difference between proton and neutron single-particle wave functions. In the present study, we assume that the charge-symmetry breaking and all other deficiencies of the WS potential can be cured by readjustment of the well depth case-by-case to reproduce experimental proton and neutron separation energies. The length parameter of the central term was determined from a condition that the charge density constructed from the proton radial wave functions yields a root-mean-charge radius in agreement with the experimental value measured by electron scattering [13] or by isotope-shift estimation [1].

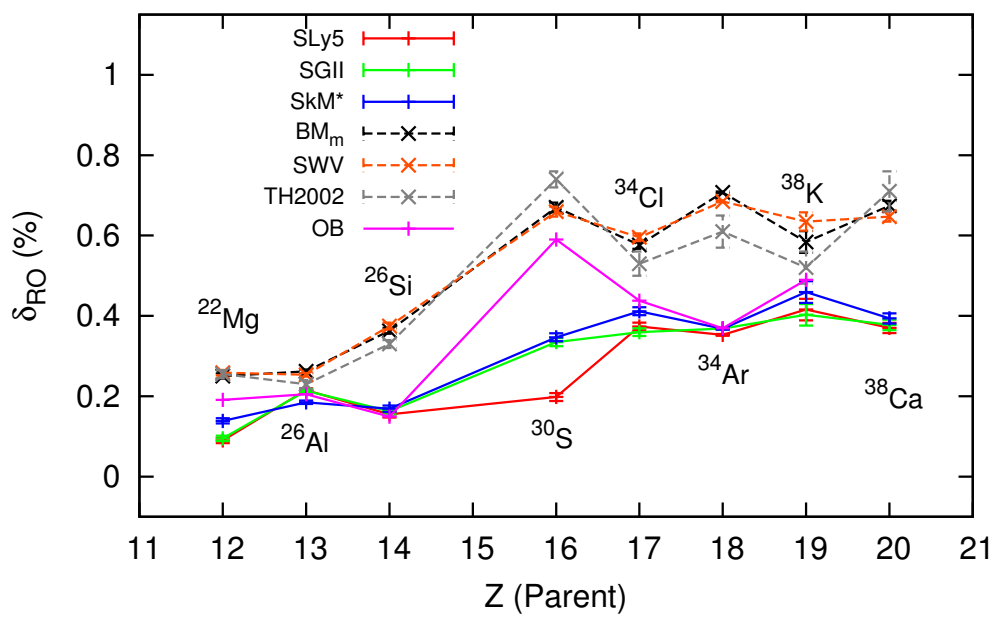

Fig. 1. (Colour on-line) Calculated values for the radial overlap correction, $\delta_{\mathrm{RO}}[\%]$ in comparison with the results of Ormand and Brown (OB) $[3,5]$ and those of Towner and Hardy (TH2002) [1].

The spherical HF calculations have been performed with three different Skyrme forces, namely, SGII [14] and SkM* [15] and SLy5 [16]. While SGII and $\mathrm{SkM}^{*}$ were already used in Ref. [3], SLy5 is a more recent parametrisation by the Saclay-Lyon collaboration. It was constructed to reproduce various bulk nuclear properties and selected properties of a number of dou- 
bly magic nuclei, without ${ }^{16} \mathrm{O}$. Since the nuclei of interest are open-shell systems, we have assumed a uniform occupation of a last occupied, partly filled orbital. We have checked that shell-model occupation numbers for initial and final $0^{+}$states, obtained from the diagonalisation, produce very similar results. The central part of the self-consistent potential of the parent and daughter nuclei was scaled in order to reproduce experimental proton and neutron separation energies, respectively. We have tested that this scaling only little influences the charge radii of nuclei considered, which stay in a very good agreement with experiment. The Coulomb exchange term was accounted within the Slater approximation. Our preliminary results [12] show that its exact calculation only marginally affects the $\delta_{\mathrm{RO}}$ value.

As is seen from the figure, all our WS results are quite close to each other, indicating that the correction $\delta_{\mathrm{RO}}$ is not very sensitive to a particular choice of the WS potential parameters. In general, they are in a fair agreement with the shell-model plus WS calculation of Towner and Hardy in 2002 [1], except for ${ }^{34} \mathrm{Ar}$ and ${ }^{38} \mathrm{~K}$ because we used new experimental data for the charge radius [13]. We do not compare our present results with the latest calculation of Towner and Hardy [6], performed with the inclusion of the orbitals outside the valence space. The work in this direction is in progress.

For the HF case, we find that the correction only little depends on a particular version of the Skyrme force, except for ${ }^{30} \mathrm{~S}$. Overall, our results are consistent with those of Ormand and Brown [5], again with the exception of ${ }^{30} \mathrm{~S}$. In the case of ${ }^{30} \mathrm{~S}$, the correction $\delta_{\mathrm{RO}}$ is dominated by the $2 s_{1 / 2}$ state, in which the centrifugal barrier is not present, and thus the radial wave function is very sensitive to the fine details of the mean field. We note that SLy5 interaction results in a considerably smaller $\delta_{\mathrm{RO}}$ value compared to SGII and SkM*.

We do not confront our results to the most recent calculation of $\delta_{\mathrm{RO}}$ with Skyrme-HF wave functions carried out by Hardy and Towner in 2009 [17]. Unlike the standard HF procedure, they performed a single calculation for the nucleus with $(A-1)$ nucleons and $(Z-1)$ protons, and then used the proton and the neutron eigenfunctions from the same calculation to compute radial integrals. Since Koopmans's theorem is not fully respected by such HF calculations, in particular, with a density-dependent effective interaction, we do not consider their protocol to be well-justifiable.

The $\delta_{\mathrm{RO}}$ values obtained with $\mathrm{HF}$ wave functions are seen to be systematically smaller than those obtained with WS wave functions. The reason can be easily understood. The Skyrme interaction is usually supposed to be isospin invariant. However, the presence of the Coulomb term causes a difference between proton and neutron densities, inducing an isovector term in the self-consistent mean-field potential $[3,18]$. That term tends to counter Coulomb repulsion, therefore reducing $\delta_{\mathrm{RO}}$. It will be interesting to study 
whether charge-symmetry (CSB) and charge-independence breaking (CIB) terms in a conventional isospin-invariant Skyrme interaction affect the value of the correction.

We are grateful to B. Blank for stimulating discussions. L. Xayavong thanks Université de Bordeaux for a Ph.D. fellowship. The work was supported by the CFT (IN2P3/CNRS, France), AP théorie 2014-2016.

\section{REFERENCES}

[1] I.S. Towner, J.C. Hardy, Phys. Rev. C 66, 035501 (2002).

[2] J.C. Hardy, I.S. Towner, Phys. Rev. C 91, 025501 (2015).

[3] W.E. Ormand, B.A. Brown, Nucl. Phys. A 440, 274 (1985).

[4] G.A. Miller, A. Schwenk, Phys. Rev. C 78, 035501 (2008).

[5] W.E. Ormand, B.A. Brown, Phys. Rev. Lett. 62, 8 (1989).

[6] I.S. Towner, J.C. Hardy, Phys. Rev. C 77, 025501 (2008).

[7] B.H. Wildenthal, Prog. Part. Nucl. Phys. 11, 5 (1984).

[8] B.A. Brown, W.A. Richter, Phys. Rev. C 74, 034315 (2006).

[9] B.A. Brown, W.D.M. Rae, Nucl. Data Sheets 120, 115 (2014).

[10] N. Schwierz, I. Wiedenhover, A. Volya, arXiv:0709.3525 [nucl-th].

[11] A. Bohr, B.R. Mottelson, Nuclear Structure, Vol. I, World Scientific, 1998.

[12] L. Xayavong, Ph.D. Thesis, University of Bordeaux, 2016.

[13] I. Angeli, K.P. Marinova, ADNDT 99, 69 (2013).

[14] N. Van Giai, H. Sagawa, Nucl. Phys. A 371, 1 (1981).

[15] J. Bartel et al., Nucl. Phys. A 386, 79 (1982).

[16] E. Chabanat et al., Nucl. Phys. A 635, 231 (1998).

[17] J.C. Hardy, I.S. Towner, Phys. Rev. C 79, 055502 (2009).

[18] C.B. Dover, N.V. Giai, Nucl. Phys. A 190, 373 (1972). 